\title{
THE RESULTS OF SIMULATING A DIGITAL IMAGING NETWORK SYSTEM IN ECHELON 3 OF DEPLOYABLE COMBAT MEDICAL FACILITIES
}

\author{
William P. Johnson, Ph.D. \\ The MITRE Corporation, W21 \\ 7525 Colshire Drive \\ McLean, Virginia 22102-3481
}

\begin{abstract}
The U.S. Army is evaluating the conversion of all of its conventional medical radiological imaging capabilities to a filmless technology using a Digital Imaging Network System (DINS) in both fixed and field hospitals. The latter hospitals are called Deployable Combat Medical Facilities. Using DINS, the photosensitive films presently used to record and display $\mathrm{x}$-ray images would be replaced by digitized images stored on either magnetic or optical devices and displayed on computer workstations (electronic lightboxes). This document describes the use of a simulation model as a tool in estimating the imaging caseload for field hospitals for the purpose of determining the required image storage capacities of the DINS workstations and the required throughput capacity of the inter-workstation local area network. The simulation follows a patient through the five echelons of field hospitals, recording the number of radiological images created in each ward of a hospital and the inter-ward movement of images. The simulation also provides information about the number of images transferred with a patient being evacuated to rear area hospitals. The detail included in the simulation model makes it possible to analyze the utilization of facilities within a hospital and the impact of queuing on patient care when these facilities are overloaded. Three simulation examples are presented that examine the impact on an Echelon 3 EVAC Hospital of the treatment of patients with combat-related injuries.
\end{abstract}

\subsection{INTRODUCTION}

The U.S. Army proposes to replace all current radiological imaging devices in the radiological departments of their medical facilities in the United States and overseas with filmless image capture, storage, and display equipment. With a filmless system, images are captured by various radiological modalities in digital form so that they can be stored, transported, displayed, and copied by a DINS consisting of a number of workstations inter-connected by a local area network (LAN). Included within the Army medical facilities are the Deployable Medical Systems (DEPMEDS), which are field hospital facilities normally maintained in storage until needed for treatment of casualties resulting from military action or natural disasters occurring anywhere in national or international theaters of operation. For field hospitals, filmless radiological imaging technology is seen as having the potential to enhance the triage and treatment process by reducing the incidence of lost $\mathrm{x}$-ray images and facilitating the movement of these images throughout the various wards of a field hospital. A compact light-weight medium, e.g., optical card or tape, can be used for transporting images between field hospital facilities. In addition, the image processing capabilities of a DINS workstation provide the potential for computer enhancement of poor quality images, reducing the need for "re-takes." An additional benefit for the military of filmless radiology is the elimination of the logistical burden of maintaining perishable film, chemical supplies, and fresh water for each field hospital.

One task that must be completed before DINS equipment can be procured is to size the storage capacities needed for the DINS workstations used in each hospital for storing, distributing, and displaying radiological images taken in the current hospital as well as images acquired in lower echelons. In support of this effort, The MITRE Corporation has developed a simulation model to predict medical image workload under various combat scenarios. This model simulates the flow of casualties through the various echelons of field hospitals to determine how many diagnostic images are generated, where they are physically generated within each hospital facility, and how often and where these images are viewed by the medical care providers. Additionally, the number of images and records that accompany casualties to higher medical care echelons and medical record archival facilities has been estimated based upon current Army doctrine. Using the simulation model developed by MITRE, input parameters can be adjusted for a particular hospital configuration with output from the model providing the information needed to size the workstation storage capabilities, the load on the inter-workstation communication network, and the inter-hospital transportable record requirements.

\subsection{THE FIELD HOSPITAL SYSTEM}

Within the U.S. Army field medical care organization, there are four echelons of hospitals that have a direct and immediate impact on the treatment and care of military patients, with Echelon 1 heing the most forward echelon. A fifth echelon, the hospitals in the Continental United States, handles the most severely injured soldiers requiring long periods of recovery (e.g., greater than 60 days) before returning to duty (RTD) or being discharged from military service for medical reasons. The configuration of hospitals within each echelon and the structure of each hospital is currently under study by the U.S. Army. However, for the purposes of performing this simulation, the organization shown in Figure 1, which illustrates the relationships between the five echelons and the patient flow through them, has been used. If one were to think of field hospitals in a Korean conflict, Echelons 1, 2, and 3 would be located in Korea, with the Echelon 4 hospital in Japan.

For a given field hospital, the DINS will consist of a number of compatible, interchangeable computer workstations. Each workstation will have one or more high resolution display screens, a mass storage device, a keyboard-type (i.e., manual) input device, a connection for a local area network, an input port for retrieval of 


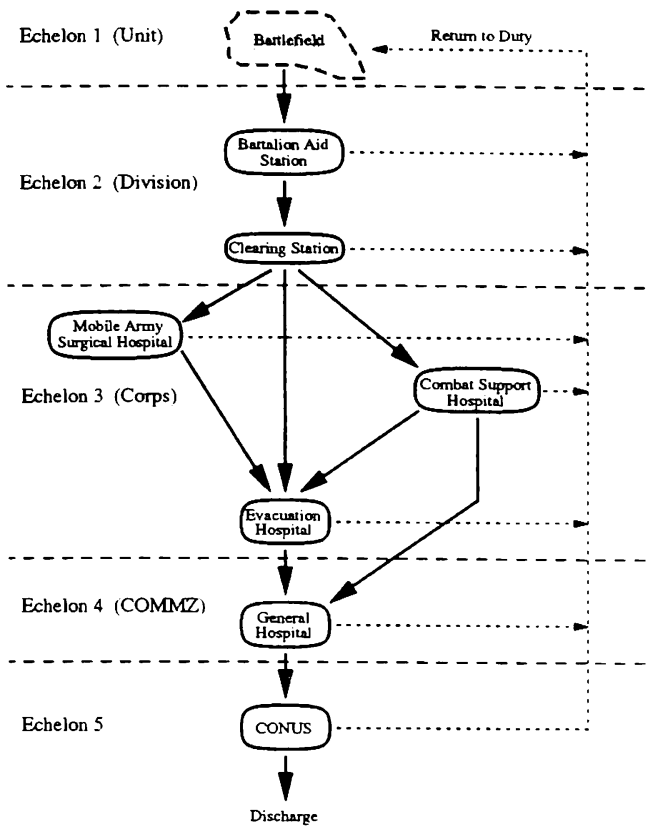

Figure 1. Casualty Flow through the Five Echelons of Combat Care

data from image generating devices, and an output port for generating copies of stored images on an optical medium for archiving or transport to other hospitals. These workstations are located throughout the hospital and are interconnected via a LAN. Figure 2 illustrates a possible deployment of DINS workstations in a field hospital.

\subsection{DESCRIPTION OF THE SIMULATION MODEL}

The simulation model developed for this analysis follows a wounded soldier from the forward area in Echelon 1 through to either transfer to Echelon 5 or return to duty. The simulation software package used for programming this model is Simulation Language for Alternative Modeling (SLAM II). The emphasis of the simulation described in this paper is on collecting information on hospitals in Echelon 3, but the model can be expanded to include a detailed simulation of hospitals in Echelons 2 and 4.

Echelon 3 is divided into three functionally and geographically separated hospitals: Mobile Army Surgical Hospital (MASH), Evacuation Hospital (EVAC), and Combat Support Hospital (CSH). The model divides a hospital into six wards:

o Emergency treatment ward (EMT)

o X-ray and computed tomography (CT) units

o Operating room (OR)

o Intensive care unit (ICU)

o Internediate care ward (ICW)

o Minimum care ward (MCW)

Assumptions are made about casualty arrival time distribution, casualty flow from Echelons 1 through 4 hospitals, image transmission within a hospital, and configuration of the hospitals within Echelon 3. For the examples presented, it is assumed that
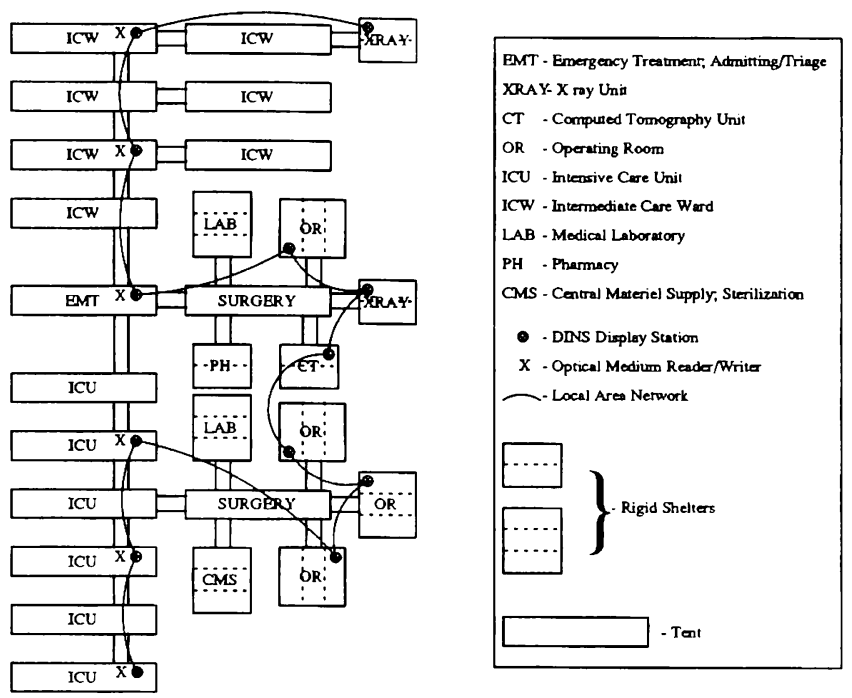

Figure 2. A Typical Deployment of DINS Workstations in a Field Hospital

the MASH Hospital is only a routing facility for sending patients to the CSH Hospital, if the predicted RTD is fewer than 30 days, or to an EVAC Hospital. The primary input data for the model consist of a list of patient conditions (PCs), which describe classes or types of injuries, the treatment processes and $x$-ray requirements for each, the time spent in each ward, and data on the relative frequency of occurrence of each PC. Additional input data consist of a hospital description (number of beds, x-ray machines, etc.), casualty arrival rates during a combat day, length of a combat day, and number of combat days.

The output generated by the simulation provides information on active image storage requirements in the individual wards and in the hospital and on inter-workstation image transfer traffic on the LAN. Data on the number of images transferred between hospitals are also collected. Within a hospital, data are collected on the delay time experienced by workstations attempting to send images over the LAN and on the size of the image files being transferred.

\subsection{SIMULATION RESULTS}

Three simulation examples are presented that examine on the impact on an Echelon 3 EVAC Hospital of the treament of patients with combat-related injuries. In the first example, combat casualties occur at a rate of 50 per day, resulting in approximately 45 casualties per day arriving at an EVAC Hospital. By the third day of conflict in this example, long queues develop in OR with waiting times averaging five hours. The second example increases the number of OR tables in this same EVAC Hospital, beginning on the third day of battle. This second example is included to illustrate how a simulation can be used as a tool in analyzing the impact of command decisions on hospital operation. The third simulation example shows the impact on DINS of adding 
computed tomography (CT) to the diagnostic tools used in an EVAC Hospital.

With the exception of the increase in OR tables in example 2, the following input parameters are used in the example simulations:

o Number of continuous battle days, 10

o Casualties per day, 50

o LAN data transfer rate (Mbits/sec), 8

o Number of EMT beds, 12

o Number of $x$-ray units, 2

o Number of CT units, 1

- Number of OR tables, 6

- Number of ICU beds, 90

o Number of ICW/MCW beds, 360

The ten-day battle input parameter was chosen merely to provide time for the conditions in the EVAC Hospital to stablize, which occurred by the third or fourth simulation day. The simulation calculation covered a 15-day time period to show how rapidly the EVAC Hospital can recover from a cessation of combat.

The operational assumption for image storage is that patient images are stored only on workstations in the hospital ward where the patient is currently located. These images are transported between workstations over the LAN. They are removed from active storage in the DINS when a patient leaves the hospital. The LAN access protocol used is equivalent to carrier sense multiple access with collisons prohibited and queued when simultaneous LAN access requests occur. The storage requirements for archival of images for permanent patient records is a policy issue that is not of concem in this document, although output from the simulations can provide the required data when an archival policy has been established.

For the first example, simulation results on the peak number of images actively stored in each EVAC Hospital ward and in the hospital as a whole are given in Table 1. Assuming that a $1024 \mathrm{x}$ 1024 pixel by 12 bits per pixel image (in a compacted format) requires 1.5 Mbytes of storage space, Table 1 also gives the peak active storage requirements. If the ward workstations are equipped with two hard disk drives each with a nominal 700 MByte capacity, even the workstation in the ICW ward can store all images for patients in this ward, assuming the workstation distribution of Figure 2. Table 1 shows that the number of inages stored in the wards is not well distributed among the wards for the

Table 1. Peak Image Storage Requirements in an EVAC Hospital

\begin{tabular}{|ccc|}
\hline $\begin{array}{c}\text { Active } \\
\text { Images } \\
\text { in } \\
\text { Wards }\end{array}$ & $\begin{array}{c}\text { Peak } \\
\text { Number of } \\
\text { lmages } \\
\text { Stored }\end{array}$ & $\begin{array}{c}\text { Peak } \\
\text { Storage } \\
\text { Requirements } \\
\text { (MBytes) }\end{array}$ \\
\hline XRAY & 20 & 30 \\
OR & 310 & 465 \\
ICU & 762 & 1,143 \\
ICW & 514 & 771 \\
Total for Hospital & 1,398 & 2,097 \\
\hline
\end{tabular}

image movement scheme used in this simulation. Other image movement schemes can be tried by modifying the model and performing new simulations. Table 2 presents results on the number of images transferred to Echelons 4 and 5 when casualties are evacuated to rear areas for further treatment and recuperation. These images can be carried with the patient on a storage medium such as an optical card or disk.

\begin{tabular}{|ccc|}
\hline Table 2. Images Transferred to Echelons 4 and 5 \\
\hline & $\begin{array}{c}\text { Number of } \\
\text { Images }\end{array}$ & $\begin{array}{c}\text { Storage } \\
\text { Requirements } \\
\text { (MBytes) }\end{array}$ \\
\hline Transferred to Echelon 4 & 3,441 & 5,162 \\
Transferred to Echelon 5 & 3,553 & 5,330 \\
\hline
\end{tabular}

Additional simulation results show that the total number of requests to send images over the LAN averaged 1,540, during the 15-day simulation, with an average of 5.8 images sent per request. The number of images sent varied from a minimum of two to a maximum of 14 per request. In order for a medical provider to be able to view an image on a workstation within a few seconds of making the request, any images transferred between workstations over the LAN must be sent at a high data rate. In the simulation, a LAN data rate of $8 \mathrm{Mbits}$ per second was used. The average time between requests for any workstation to send images over the LAN is 12 minutes, which is a low relative data throughput requirement for a LAN with such a high data rate, and contention for access to the LAN should be rare. However, occasionally, the simulation found that the LAN was busy when a request to send images was made, causing a transmission delay. The simulation output indicated that 99 percent of the time a workstation encountered a transmission delay of less than one-half second (the minimum resolution of the measured delay), with a maximum delay greater than one second occurring only 13 times out of the total of 1,540 requests made. The longest delay was 11 seconds. Since the LAN used in the model closely resembles a $10 \mathrm{Mbits}$ per second Ethernet, this type of LAN should easily satisfy the inter-workstation communication needs for the hospital in this example.

Figure 3 illustrates how hospital beds and OR tables are utilized during the 15-day simulation period for the first example. This figure shows that the six OR tables are very heavily utilized. Further examination of the simulation output shows the average waiting time in the OR queue is nearly five hours. Figure 4 is a histogram of these waiting periods and illustrates that approximately 20 percent of the casualties face waits exceeding ten hours. The response of a commander to this deterioration in medical care might be to move an additional number of OR units to the hospital. A change was made to the simulation program in the furst example that increased by four the total number of available OR tables, 48 hours after the battle begins. The impact of such a change was simulated, as example 2, and the effects on the OR queuing problem are illustrated in Figure 5, which shows a dramatic reduction in OR waiting times. 


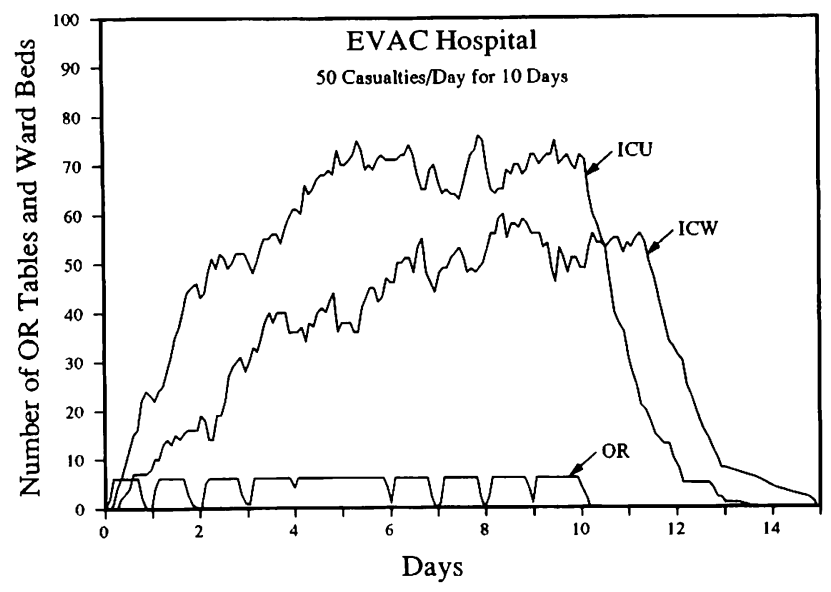

Figure 3. Utilization of OR Tables and Ward Beds

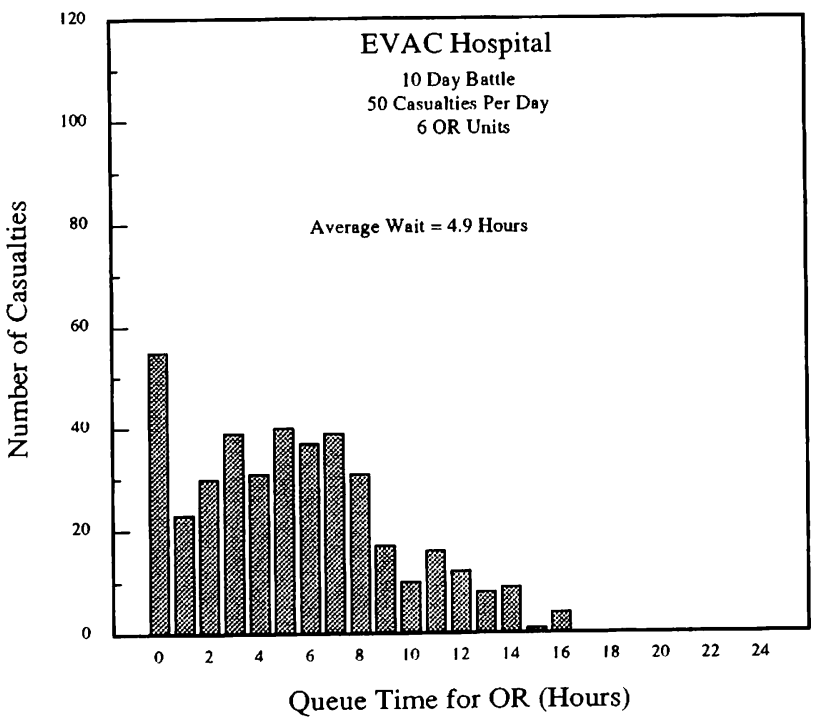

Figure 4. Histogram of Operating Room Queue Waiting Times
For the third example, the input data have been modified to include the use of computed tomography exams for certain types of injuries. These CT exams generate images that have great diagnostic value but require a large amount of digital storage capacity. For this simulation, it is assumed that the storage requirements for a CT exam are ten times that of a single $\mathrm{x}$-ray (i.e., $40 \mathrm{CT}$ slices at a resolution of $512 \times 512$ pixels by 12 bits per pixel). Table 3 compares the peak storage requirements for $x$-xays only with the requirements for $\mathrm{x}$-rays plus CT exams, and Table 4 compares the effect on number of images accompanying evacuees to Echelons 4 and 5. The introduction of CT diagnostic imaging in an EVAC Hospital increases the peak storage requirements by about 40 percent; even so, the bulk storage capabilities of the hard disk drives on the workstations can still accommodate the added load.

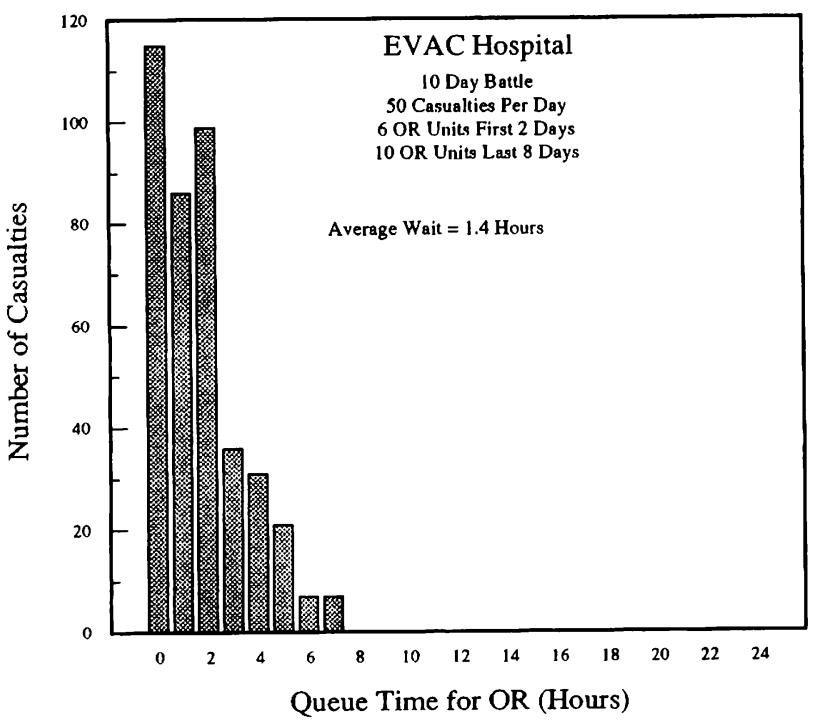

Figure 5. Histogram of Operating Room Queue Waiting Times with Increase in OR Tables

\begin{tabular}{|ccc|}
$\begin{array}{c}\text { Table 4. Comparison of Images Transferred to Echelons } 4 \text { and } 5 \\
\text { for X-rays Only and for X-rays Plus CTs }\end{array}$ & \multicolumn{2}{c|}{ Images (MBytes) } \\
\hline & $\begin{array}{c}\text { X-rays } \\
\text { Only }\end{array}$ & $\begin{array}{c}\text { X-rays } \\
\text { Plus CTs }\end{array}$ \\
\hline Transferred to Echelon 4 & 5,162 & 7,119 \\
Transferred to Echelon 5 & 5,330 & 7,515 \\
\hline
\end{tabular}

Table 3. Comparison of Peak Storage Requirements for X-rays Only and for X-rays Plus CTs

\begin{tabular}{|ccc|}
\hline Active & \multicolumn{3}{c|}{ Stored Images (MBytes) } \\
\cline { 2 - 3 } Images & X-rays & $\begin{array}{c}\text { X-rays } \\
\text { in }\end{array}$ \\
Wards & Only & Plus \\
\hline XRAY & 30 & 83 \\
OR & 465 & 723 \\
ICU & 1,143 & 1,791 \\
ICW & 771 & 1,080 \\
Total for Hospital & 2,097 & 3,074 \\
\hline
\end{tabular}




\subsection{CONCLUSIONS}

A simulation model has been developed to analyze the movement of combat casualties through the field hospital medical system with the purpose of obtaining data relevant to sizing the storage requirements of DINS workstations and the inter-workstation LAN within a hospital. The program and examples presented in this document emphasize an EVAC Hospital in Echelon 3.

The examples cited in this document indicate that for the EVAC Hospital, the workstation bulk storage requirements for active images are achievable with existing magnetic and optical storage technology, even if CT diagnostic imaging is introduced. Also, the inter-workstation communication of image data places no throughput burden on a LAN such as a $10 \mathrm{Mbit} / \mathrm{sec}$ Ethernet.

Simulations of the movement of patients through a combat field hospital system can be performed quickly by the MTRE-developed model used for the examples in this paper. This simulation model has the flexibility to accommodate changes in hospital organization, patient arrival rate, and type and frequency-of-occurrence of injuries by modifying program variables. In a few hours, it is possible to simulate several alternative configurations for various combat situations.

\section{ACKNOWLEDGEMENTS}

This work is supported by the U.S. Army Medical Research Acquisition Activity, Contract No. DAMD17-86-C-6145. The views, opinions, and/or findings contained in this report are those of the author and should not be construed as an official Department of the Army position, policy, or decision unless so designated by other documentation.

\section{AUTHOR'S BIOGRAPHY}

WILLIAM P. JOHNSON is a Member of the Technical Staff in the Information and Telecommunications Networks Department of The MITRE Corporation, where he is currently involved in developing and using simulation models to analyze the workstation storage capacities and LAN requirements for DINS in Army field hospitals. He also is participating in the development of a proof-of-concept DINS prototype currently being demonstrated in military field hospitals. He received a BS and MS in Physics and a Ph.D. in Experimental Nuclear Physics from Indiana University.

William P. Johnson

The MITRE Corporation

7525 Colshire Drive, M/S Z565

McLean, VA, 22102-3481 